\title{
Chronic visceral ischemia: An unusual cause of abdominal pain
}

\author{
Turan Acar $^{1}$ (D), Volkan Çakır (D), Nihan Acar ${ }^{1}$ (D), Kemal Atahan ${ }^{1}$ (D), Mehmet Hacıyanlı (D)
}

\section{ABSTRACT}

ORCID IDs of the authors:

T.A. 0000-0003-4261-2673;

V.C. $0000-0002-3039-8262 ;$

N.A. $0000-0003-0720-3794$;

K.A. 0000-0002-0096-8789;

M.H. 0000-0002-0512-1405.

Cite this paper as:

Acar T, Çakır V, Acar N,

Atahan K, Haciyanlı M.

Chronic visceral ischemia: An

unusual cause of abdominal

pain. Turk J Surg 2018; 34:

158-161.

'Department of General Surgery, İzmir Katip Çelebi University Atatürk Training and Research Hospital, İzmir, Turkey 2Department of Interventional Radiology, İzmir Katip Çelebi University Atatürk Training and

Research Hospital, İzmir, Turkey

This study was presented at the $19^{\text {th }}$ National Surgical Congress,

16-20 April 2014, Antalya, Turkey.

\section{Corresponding Author}

Turan Acar

e-mail:drturanacar1982@gmail.com

Received: 17.05.2015

Accepted: 30.08 .2015

Available Online Date: 03.01.2018

OCopyright 2018

by Turkish Surgical Association

Available online at

www.turkjsurg.com
Chronic visceral ischemia is described as postprandial abdominal pain caused by intestinal hypoperfusion. Chronic visceral ischemia arising from the stenosis of major mesenteric arteries can cause death. Chronic abdominal pain, weight loss, and sitophobia are the major symptoms. The main cause of chronic visceral ischemia is atherosclerosis; Doppler ultrasonography, tomographic angiography, and magnetic resonance angiography can be used for diagnosis. The gold standard method is mesenteric catheterized angiography. Surgical bypass or endovascular balloon angioplasty and stent replacement can also be performed to prevent serious complications and death. A total of three patients, two male and one female, applied to emergency services with blunt abdominal pain lasting a few hours that started after meals and was located in the epigastric and periumblical regions. The patients were diagnosed with chronic visceral ischemia after screening tests and physical examination. Mesenteric catheterized angiography was performed immediately in all the cases. Balloon angioplasty and stent replacement were performed on the stenoses, and occlusions were detected. Mesenteric catheterized angiography may be preferred in cases with strong clinical suspicion; balloon angioplasty and stent replacement can also be used as treatments with lower rates of complications.

Keywords: Chronic mesenteric ischemia, superior mesenteric arteries, balloon angioplasty, stent

\section{INTRODUCTION}

Chronic mesenteric ischemia (CMI) is described as postprandial abdominal pain caused by intestinal hypoperfusion. $\mathrm{CMI}$ is a rare condition that is caused by stenosis of the major mesenteric arteries and can be fatal; it was first described by Goodman as "abdominal angina" in 1918. CMI has a mostly multifactorial etiology; it is the most common cause of atherosclerosis in the celiac artery (CA), superior mesenteric artery (SMA), and inferior mesenteric artery (IMA) (1). CMI arising from stenosis of the major mesenteric arteries can cause death. However, arterial dissection can occur due to vasculitis, fibromuscular dysplasia, radiation, and use of cocaine. General risk factors are smoking, hypertension, diabetes mellitus (DM), and hypercholesterolemia.

The clinical course progresses slowly and is difficult to diagnose. The major symptoms are 15 to 60 minutes of abdominal pain starting 1 to 4 hours after eating and weight loss. Most patients are over 60 years of age, and CMI is seen three times more often in women than in men. In a study, patients over 65 years of age were found to represent $17.5 \%$ of cases with more than $70 \%$ obstruction of the mesenteric arteries (2). Chronic mesenteric ischemia can cause intestinal infarction and death if not treated. The main cause of CMI is atherosclerosis; Doppler ultrasonography (US), tomographic angiography (CTA), and magnetic resonance angiography (MRA) can be used for diagnosis. The gold standard method for accurate diagnosis is mesenteric catheterized angiography (3). The first successful open surgical procedure for CMI was performed by Shaw in 1985. Since then, surgical repair has been used as a standard treatment for CMI. However, endovascular revascularization (ER) is currently becoming more popular because ER is minimally invasive and also has lower rates of morbidity and mortality than angioplasty and stent of the mesenteric artery (4). Herein, we report three cases of CMI who presented with abdominal pain and our approach to treatment.

\section{CASE PRESENTATION}

The clinical and morphological data of the patients are summarized in Table 1. A total of three patients, two male and one female, applied to emergency services with blunt abdominal pain that started after meals, lasted a few hours, and was located in the epigastric and periumblical regions. The mean age of the patients was 50.3. The male patients had a history of smoking of one pack a day, DM, hypertension, coronary artery disease, and chronic obstructive lung disease. The female patient did not smoke and did not have a history of any other medical conditions. The patients' vital signs were stable.

In physical examination, the abdominal quadrants were sensitive to palpation, while no defense or rebound was observed. Only elevated D-dimer and fibrinogen levels were obtained in laboratory tests. All 
Table 1. Clinical and morphological findings of the patients

\begin{tabular}{|lccc} 
& Patient $\mathbf{1}$ & Patient 2 & Patient 3 \\
\hline Sex & Male & Male & Female \\
\hline Age & 57 & 45 & 49 \\
\hline Comorbidity & CAD, DM, HT, COPD, CVE & CAD, DM, HT & - \\
\hline Pathology & SMA occlusion & SMA occlusion & SMA occlusion \\
\hline Treatment & Balloon angioplasty and stenting & Balloon angioplasty and stenting & Balloon angioplasty and stenting \\
\hline Postoperative treatment & Clopidogrel 75 mg+ASA 100 mg & Clopidogrel 75 mg+ASA 100 mg & Clopidogrel 75 mg+ASA 100 mg \\
\hline Postoperative complication & Portal vein thrombosis and pancreatitis & - & - \\
\hline Hospitalization time (days) & 14 & 10 & 7 \\
\hline Follow-up time (months) & 12 & 12 & 4
\end{tabular}

Mortality

CAD: coronary artery disease; DM: diabetes mellitus; HT: hypertension; COPD: chronic obstructive pulmonary disease; CVE: cerebrovascular events; ASA: acetylsalicylic acid

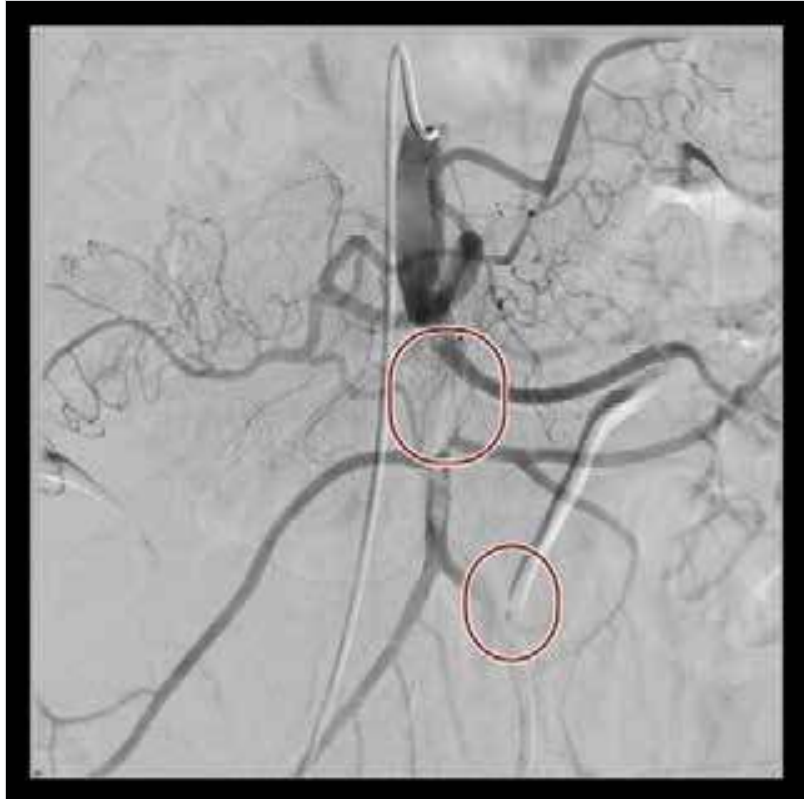

Figure 1. Angiography showing SMA and sigmoidal artery occlusion (marked with circle)

patients were investigated with abdominal contrast-enhanced computerized tomography, revealing a thrombus in the SMA. The patients were diagnosed with CMI after screening tests and physical examination. The patients were diagnosed with $\mathrm{CMI}$ due to the obliteration of the splanchnic artery, and revascularization of SMA was planned for treatment. ER, which is a minimally invasive method, was chosen due to its lower morbidity and mortality rates. Mesenteric catheterized angiography was performed immediately in all the cases. Mesenteric catheterized angiography was performed under local anesthesia in our Department of Interventional Radiology. Two of the cases had occlusions in the SMA, while the third patient had two different obstructions in the SMA and sigmoidal artery (Figure 1). Balloon angioplasty (PTA) and a $5 \mathrm{~mm}$ stent replacement was performed for each occlusion (Figure 2), and angiographic images taken after the procedure showed no obliteration (Figure 3). Balloon angioplasty and stent replacement were performed on each detected stenosis and occlusion.

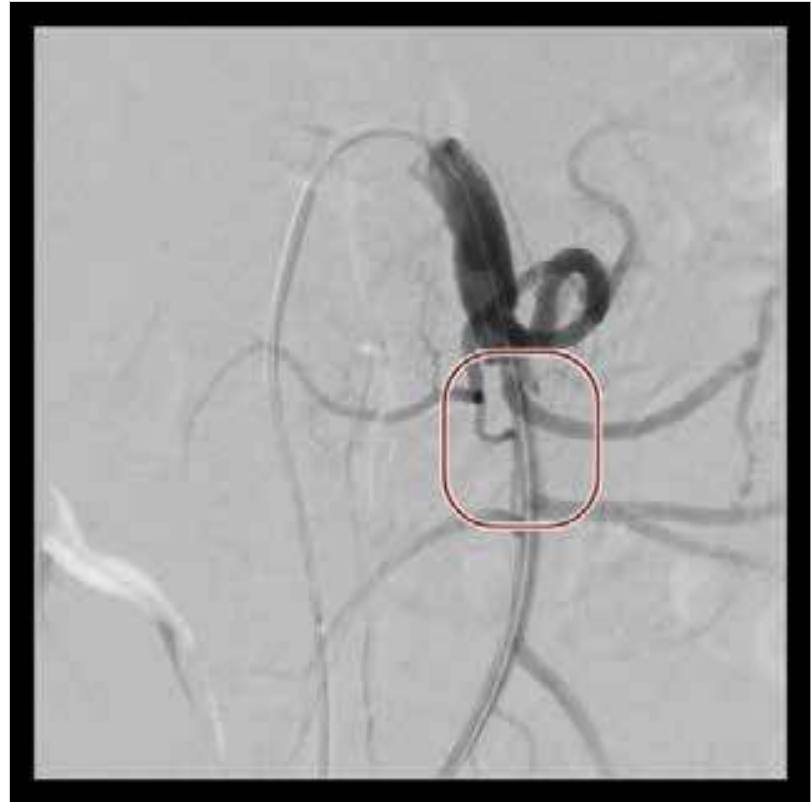

Figure 2. A $5 \mathrm{~mm}$ stent is placed in the SMA with the guidance of a wire (marked with circle)

All patients were heparinized with $500 \mathrm{U}$ before the procedure, and Clopidogrel $75 \mathrm{mg}$ and acetylsalicylic acid (ASA) were given after the procedure. During follow-up, one patient had pancreatitis and thrombosis of the portal vein and was medically treated. The other two cases had no additional complications. All three patients under follow-up remained free of disease. All the patients in this study were informed about the procedures in detail and signed informed consent forms. The necessary consent was obtained from the patients to perform a scientific study.

\section{DISCUSSION}

Chronic mesenteric ischemia, or abdominal angina, is characterized by postprandial abdominal pain and weight loss due to the loss of intestinal blood flow secondary to obstructions in two or three splanchnic vessels. CMI, which is commonly caused by atherosclerosis, is one of the less common causes of mesenteric ischemia, with a rate of $5 \%$. Other causes of CMI 


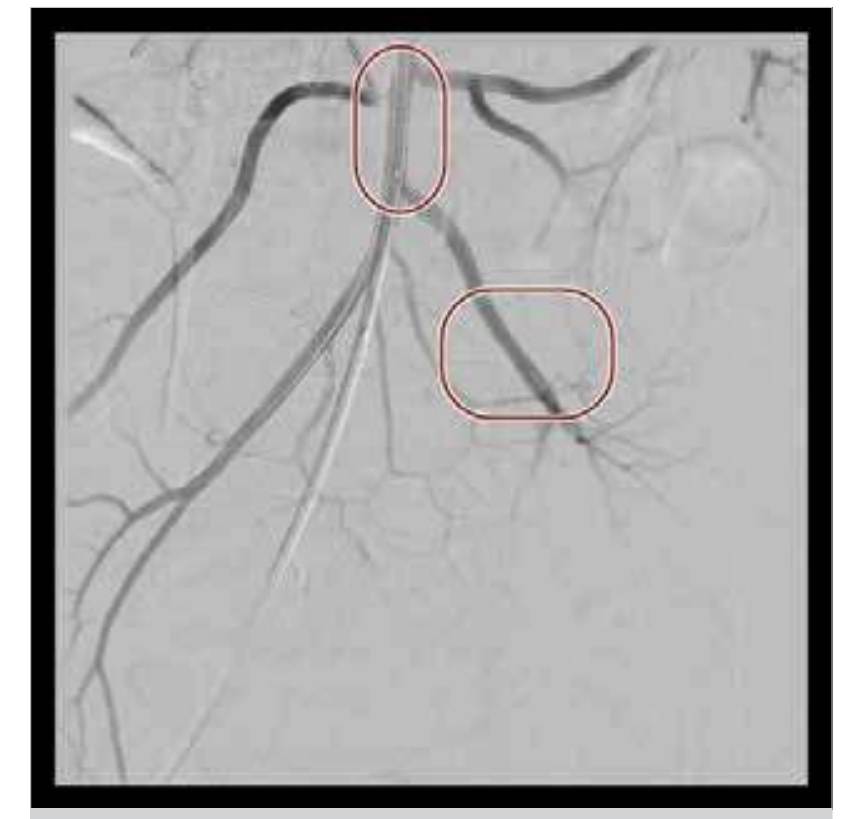

Figure 3. Open flow in the SMA and the sigmoidal artery (marked with circle)

include fibromuscular dysplasia, vasculitis (Takayasu's arteritis, giant cell arteritis, polyarteritis nodosa, systemic lupus erythematosus, and thromboangiitis obliterans), malign tumors, and radiation. The major risk factors are hypertension (57\%), cardiovascular disease (51\%), renal failure or nephropathy (30\%), and diabetes (25\%). Two of our patients had comorbid diseases of hypertension, DM, and coronary artery disease; CMI was thought to evolve due to atherosclerosis in these cases. The mean age of CMI patients is reported as 68 , and the incidence is similar between male and female patients (5).

The blood flow in the intestines is approximately $25 \%$ in hunger; however, after meals, the blood flow increases to $35 \%$. This explains why the symptoms of CMI intensify after eating. In chronic ischemia, because of the well-developed collateral circulation, symptoms usually arise only if at least two major arteries (CA, SMA, and IMA) are obstructed. Most cases remain asymptomatic, although they show more than $50 \%$ occlusion. Chronic mesenteric ischemia results in acute ischemia and bowel infarction in $26 \%-66 \%$ of untreated cases (6). Mesenteric ischemia develops in $6 \%$ of asymptomatic cases with serious mesenteric obstruction during a mean followup of 2.6 years.

Because of the gastrointestinal symptoms, mesenteric ischemia, and the mortality rate of $86 \%$ in these cases, CMI patients should be closely followed up even if they are asymptomatic. Anamnesis and physical examination are important factors in the diagnosis of CMI. Colored Doppler ultrasonography, MRA, and computerized tomographic angiography can be used as additional diagnostic techniques. A noncontrast CT examination to assess the presence of vascular calcifications should always be included. CT may also demonstrate nonvascular causes of abdominal pain, such as inflammatory disease or neoplasm. Furthermore, there may be findings that help differentiate between an acute and a chronic process; bowel wall thickening, pneumatosis, and portomesenteric gas indicate an acute process and are absent in CMI. Conventional angiogra- phy is the gold standard method for screening the main artery and branches of the mesenteric artery, in addition to stent replacement treatment and balloon angioplasty if stenosis is detected (7).

Treatments intended to address symptoms, especially postprandial abdominal pain, and to prevent bowel infarctions are necessary for symptomatic CMI patients. The initial conservative approach should include smoking cessation, bowel rest, and vasodilatator drugs. Some patients can benefit from nitrates for a short time. In cases where conservative methods fail, revascularization should be the next step. The procedure of thromboendarterectomy of the SMA was first performed by Mikkelsen in 1958. All open surgical procedures, such as transartic endarterectomy, reimplantation directly into the aorta, and antegrade or retrograde bypass, have advantages and disadvantages. The most commonly applied method is prosthetic graft bypass. A high rate of success (90\%-100\%) of surgical methods in reduction of symptoms has been reported in the literature (8). However, the mortality and morbidity of surgical procedures were stated to be $5 \%-12 \%$ and $5 \%-30 \%$, respectively (8). In addition to complications such as ileus, bleeding, intravascular coagulation, and lung, kidney, or liver failure, recurrence of the stenosis was detected in $9 \%-35 \%$ of cases.

Endovascular revascularization was first described in 1980 as an alternative to surgery. Balloon angioplasty and, if needed, stent replacement are the major components of this technique. ER, an effective and less invasive method, has become more popular due to its high rates of technical (90\%-100\%) and clinical success (80\%-100\%) in addition to the lower risk of complications (9). Additionally, the risk of open wound is lower in ER than in open surgery. Considering all these reasons, ER is currently recommended, especially for high risk patients. We also obtained satisfying results after ER treatment in our cases.

The most suitable lesions for ER are those with short segments (lower than $10 \mathrm{~cm}$ ) located near the CA or the SMA ostium. The rate of success was found to be $95 \%$ in ostial and $78 \%$ in nonostial lesions, with acceptable success of angioplasty in short term follow-up. Single vessel angioplasty is recommended in the literature; however, intervention to other vessels may be necessary in case of failure of the first attempt. SMA angioplasty was reported as sufficient in the first step. Many studies state that stent replacement after angioplasty is preferable in ostial lesions; however, it is essential in dissection and recanalization, with a decrease in the risk of recurrence.

The relative contraindications of ER include acute ischemia, bowel necrosis, acute stenosis excluding ligament compression stenosis, and advanced vascular disease affecting the secondary branches. These cases are more suitable for surgical treatment; however, the complications of perforation, bleeding, and hematoma during the procedure and postoperative distal embolization and arterial dissection may be encountered (10).

\section{CONCLUSION}

Colored Doppler ultrasonography is a useful diagnostic method for CMI. However, the assessment can be suboptimal due to diffuse abdominal distension. Mesenteric catheterized angiography may be preferred in cases with strong clinical sus- 
picion; it can also allow balloon angioplasty and stent replacement as treatments with lower complication rates.

Currently, due to increases in experience and in the technical quality of the equipment used, ER is much more suitable for the diagnosis and treatment of CMl, especially in high risk patients.

Informed Consent: Written informed consent was obtained from patient who participated in this study.

Peer-review: Externallypeer-reviewed.

Author Contributions: Concept - T.A., N.A., M.H.; Design - T.A., N.A., M.H.; Supervision - T.A., N.A., K.A.; Resource - T.A., V.C.; Materials - T.A., N.A., K.A.; Data Collection and/or Processing - T.A., N.A., K.A.; Analysis and/or Interpretation - V.C., K.A., M.H.; Literature Search - T.A., N.A.; Writing T.A., N.A. Manuscript - T.A., N.A.; Critical Reviews - K.A., M.H., V.C.

Acknowledgements: The authors would like to thank Department of General Surgery and Gastroenterology staff for their cooperation.

Conflict of Interest: The authors have no conflicts of interest to declare.

Financial Disclosure: The authors declared that this study has received no financial support.

\section{REFERENCES}

1. Sreenarasimhaiah J. Chronic mesenteric ischemia. Best Pract Res Clin Gastroenterol 2005; 19: 283-295. [CrossRef]
2. Hansen KJ, Wilson DB, Craven TE, Pearce JD, English WP, Edwards MS, et al. Mesenteric artery disease in the elderly. J Vasc Surg 2004; 40: 45-52. [CrossRef]

3. Minko $P$, Groesdonk H, Stroeder J, Miodek J, Graeber S, Bücker A et al. A scoring system for the assessment of angiographic findings in non-occlusive mesenteric ischemia (NOMI). Fortschr Röntgenstr 2012; 184: 805-809. [CrossRef]

4. Van Petersen AS, Kolkman JJ, Beuk RJ, Huisman AB, Doelman CJ, Geelkerken RH. Multidisciplinary Study Group of Splanchnic Ischemia. Open or percutaneous revascularization for chronic splanchnic syndrome. J Vasc Surg. 2010; 13: 13091316. [CrossRef]

5. Korotinski S, Katz A, Malnick SD. Chronic ischaemic bowel diseases in the aged-go with the flow. Age Ageing 2005; 34: 1016. [CrossRef]

6. Kolkman JJ, Mensink PB, van Petersen AS, Huisman AB, Geelkerken $\mathrm{RH}$. Clinical approach to chronic gastrointestinal ischaemia: from 'intestinal angina' to the spectrum of chronic splanchnic disease. Scand J Gastroenterol Suppl 2004; 241: 9-16. [CrossRef]

7. Ujiki M, Kibbe M R. Mesenteric ischemia. Perspect Vasc Surg Endovasc Ther 2005; 17: 309-318. [CrossRef]

8. Atkins M D, Kwolek C J, LaMuraglia G M, Brewster D C, Chung T K, Cambria RP. Surgical revascularization versus endovascular therapy for chronic mesenteric ischemia: a comparative experience. J Vasc Surg 2007; 45: 1162-1171. [CrossRef]

9. Kougias P, El Sayed H F, Zhou W, Lin P H. Management of chronic mesenteric ischemia. The role of endovascular therapy. J Endovasc Ther 2007; 14: 395-405. [CrossRef]

10. Zerbib P, Lebuffe G, Sergent-Baudson G, Chamatan A, Massouille $D$, Lions $C$, et al. Endovascular versus open revascularization for chronic mesenteric ischemia: a comparative study. Langenbecks Arch Surg 2008; 13: 865-870. [CrossRef] 